\title{
Preparación y caracterización de películas fotoluminiscentes de PVA dopadas con complejos metal-orgánicos de $\mathrm{Eu}^{3+}$ \\ Preparation and characterization of photoluminescent PVA films doped with metal- organic complex of Eu3+
}

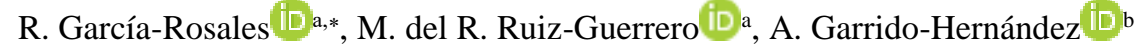 \\ ${ }^{a}$ Centro de investigación e innovación tecnológica, Instituto Politécnico Nacional, 02250, Azcapotzalco, Ciudad de México, México. \\ ${ }^{b}$ Universidad tecnológica de Tecámac, 55740, Tecámac, Estado de México, México.
}

\begin{abstract}
Resumen
El presente trabajo se centra en la caracterización de una matriz polimérica de alcohol polivinílico (PVA), preparada a partir de la dispersión de un complejo mononuclear organometálico a base de europio trivalente $\left(\mathrm{Eu}^{3+}\right)$. Los complejos de $\mathrm{Eu}^{3+} \mathrm{se}$ sintetizaron previamente en medio acuoso a condiciones ambientales empleando aniones benzoato como ligandos orgánicos, que sigue a la fórmula $\mathrm{Eu}\left(\mathrm{OOCC}_{6} \mathrm{H}_{5}\right)_{3} \cdot\left(\mathrm{H}_{2} \mathrm{O}\right)_{3}$ (Para fines prácticos la identificaremos como EuL3). Una vez caracterizado el complejo obtenido en forma de polvo, se dispersó mediante ultrasonido en la matriz de PVA. Se prepararon y caracterizaron películas a diferentes proporciones molares del complejo en relación con el PVA. Las películas fueron obtenidas mediante la técnica "Tape-casting ". Las caracterizaciones se realizaron mediante la técnica FT-IR y espectroscopia de fluorescencia, la caracterización luminiscente sugiere que la matriz de PVA genera una sensibilización en la excitación del complejo EuL3 presentando un desplazamiento en el espectro de excitación de 290 a $280 \mathrm{~nm}$; además, se observó un aumento en la emisión luminiscente debido a procesos de transferencia de energía intra o intermolecular de la matriz hacia los núcleos activos de europio.
\end{abstract}

Palabras Clave:

Luminiscencia, Complejos, Ligandos, Efecto antena, Alcohol polivinílico

\begin{abstract}
The present work focuses on the characterization of polyvinyl alcohol (PVA) polymeric matrix prepared from the dispersion of an organometallic mononuclear complex based on trivalent europium, $\mathrm{Eu}^{3+}$. The $\mathrm{Eu}^{3+}$ complexes were previously synthesized in an aqueous medium at ambient conditions using benzoate anions as organic ligands $\mathrm{Eu}\left(\mathrm{OOCC}_{6} \mathrm{H}_{5}\right)_{3} \cdot\left(\mathrm{H}_{2} \mathrm{O}\right)_{3}($ For practicality identified as EuL3). Once the complex obtained in powder form was characterized, it was ultrasonically dispersed in the PVA matrix. Films with different molar ratios of the complex to the host polymer were prepared and characterized. The Films were obtained by the "Tapecasting" technique. Characterizations were performed by FT-IR and fluorescence spectroscopy. The PVA matrix generates a sensitization in the excitation of the $\mathrm{Eu}\left(\mathrm{OOCC}_{6} \mathrm{H}_{5}\right)_{3} \cdot\left(\mathrm{H}_{2} \mathrm{O}\right)_{3}$. complex presenting a shift in the excitation spectrum from 290 to $280 \mathrm{~nm}$; in addition, an increase in the luminescent emission was observed due to intra- or intermolecular energy transfer processes from the matrix to the active europium nuclei.
\end{abstract}

Keywords:

Luminescence, Complexes, Ligands, Antenna effect, PVA

\section{Introducción}

Hoy en día los materiales que contiene iones lantánidos (Ln) dentro de su estructura son de gran interés tecnológico en diversos campos tales como: materiales ópticos, sistemas optoelectrónicos, bio-marcadores, imagenología, entre otros. Debido a que estos materiales presentan bandas de emisión estrechas e intensas en colores de alta pureza y desplazamientos Stokes significativos; sin embargo, estos materiales exhiben coeficientes de absorción relativamente bajos debido a las transiciones $\mathrm{f}-\mathrm{f}$, prohibidas por la regla de selección de La Porte (Binnemans, 2015). Por lo antes mencionado, algunos trabajos señalan que estos iones requieren el uso de sensibilizadores energéticos que promuevan sus propiedades ópticas (LITTELL WC \& JR, 1968).

La literatura reporta el uso de moléculas orgánicas como agentes acomplejantes o ligandos para la síntesis de complejos de coordinación con núcleos lantánidos (Andreiadis y col. 2013; Byrne y col.2015). Estos agentes acomplejantes generan el

\footnotetext{
*Autor para la correspondencia: rgarciar1903@alumno.ipn.mx Guerrero).

Correo electrónico: rgarciar1903@alumno.ipn.mx (Roberto García-Rosales) agarridoh@uttecamac.edu.mx (Aristeo Garrido-Hernández), maruizg@ipn.mx (María del Rosario Ruiz- 
fenómeno denominado "efecto antena", el cual consiste en concentrar la energía lumínica hacia los núcleos lantánidos. Este fenómeno es un proceso de conversión de luz a través del cual se genera una secuencia de absorción-transferencia-emisión de energía, donde los ligandos funcionan como colectores de luz y el ion lantánido como un emisor de energía en forma de fotones (Pham y col.2017). La luminiscencia sensibilizada de un complejo de lantánido implica: la absorción de luz por parte del ligando, el cruce entre sistemas desde el estado excitado singlete al estado triplete, la transferencia de energía del estado triplete al ion lantánido y la emisión de luz por parte del lantánido (Crosby y col.1961).

Los ligandos no solo sirven como antena para sensibilizar el ion lantánido, sino que también lo protege de ambientes que propicien la desactivación fluorescente o "efecto quenching". Por lo tanto, la elección del ligando requiere de una estrategia de diseño especial que cumpla con los siguientes puntos: el ligando debe 1) poseer estados de donación de energía adecuados para una transferencia de energía eficiente, 2) excluir la coordinación de disolventes y 3) eliminar otras vías de desactivación, como los estados de transferencia de carga del ligando al metal (LMCT) y las vibraciones $\mathrm{O}-\mathrm{H}$ y $\mathrm{N}-\mathrm{H}$, además de que se deben generar complejos altamente estables, si se quieren utilizar en solución y/o en dopaje de películas poliméricas (Chen y col. 2018). Los complejos organometálicos sintetizados con núcleos de europio son los más estudiados actualmente ya presentan bandas de emisión situadas a 578, 592, 613, 650 y $700 \mathrm{~nm}$ correspondientes a las transiciones ${ }^{5} \mathrm{D}_{0}-{ }^{7} \mathrm{~F}_{\mathrm{J}}(\mathrm{J}=0,1,2,3,4)$. Como el estado excitado ${ }^{5} \mathrm{D}_{0}$ se encuentra en $17200 \mathrm{~cm}^{-1}$, el nivel de energía donante de los ligandos debe ser de al menos $19200 \mathrm{~cm}^{-1}(2000$ $\mathrm{cm}^{-1}$ más alto) para una transferencia de energía eficiente. Los ligandos de ácidos carboxílicos y piridina-2,6-dicarboxílico son de los más comunes (Xiang y col. 2017).

Jing Zhang y col. (2013) sintetizaron una serie de complejos de europio con diferentes ligandos de ácidos carboxílicos y fenantrolina (phen), los cuales se comprenden dentro de la siguiente formula $\mathrm{Eu}(\mathrm{Lc})_{3}$ phen $(\mathrm{Lc}=$ ácido metacrilico $(\mathrm{MAA})$, ácido acético (AA), ácido benzoico (BA), ácido salicílico (SA) en donde demostraron las diferencias en las propiedades luminiscentes de los distintos complejos mismas que son causadas por las diferentes estructuras de los ligandos. Observaron que la intensidad de absorción de los ligandos de ácido benzoico fue la más fuerte, seguida por MAA y AA y SA la cual fue más débil. Por lo tanto, la intensidad de fluorescencia de $\mathrm{Eu}(\mathrm{BA})_{3}$ phen fue la más fuerte, seguida por $\mathrm{Eu}(\mathrm{MAA})_{3}$ phen, $\mathrm{Eu}(\mathrm{AA})_{3}$ phen $\mathrm{y}$ $\mathrm{Eu}_{2}(\mathrm{SA})_{3}$ phen $_{2}$, esta última siendo la más débil, de esta manera evidenciaron que el efecto por impedimento estérico y por coincidencias de nivel entre los ligandos de ácido carboxílico y el ligando heterocíclico, tienen un impacto significativo en la eficiencia de absorción de energía de los complejos. Además, indicaron que los complejos de $\mathrm{Eu}^{3+}$ tienen gran potencial como materiales de emisión orgánica de base molecular en la tecnología OLED ya que su emisión en color rojo es importante para los sistemas de color RGB.(Zhang y col.2013).

Como ya se mencionó, los complejos de coordinación de Ln se pueden utilizar para preparar materiales híbridos complejopolímeros, los cuales presentan excelentes propiedades mecánicas y fotofísicas que pueden extender el uso de complejos lantánidos hacia películas fotoconversoras, tintas antifalsificación e iluminación fría (Knyazev y col. 2017).

Actualmente se han reportado distintos trabajos que evidencian una mejora sustancial en propiedades físicas, mecánicas y ópticas de complejos de elementos lantánidos al ser dispersados en matrices poliméricas(Gil-Kowalczyk y col. 2021). De esta manera se tienen sistemas compuestos derivados de la combinación de las propiedades ópticas de los complejos de lantánidos y las propiedades físicas de algunos polímeros, Los polímeros pueden ofrecer estabilidad termoquímica, flexibilidad, protección ante ambientes oxidantes, e incluso confiriéndoles biocompatibilidad. (Regalado-Pérez y col.2020; Sahoo y col. 2017).

Entre las matrices más utilizadas y hasta ahora reportadas para la formación de estos sistemas poliméricos son el polimetilmetacrilato (PMMA), polímeros fluorados, poli- $\beta$ hidroxibutirato (PHB), etilvinilacetato (EVA) y alcohol poli vinílico (PVA) (Gibelli et al., 2013; Gross y col. 2007; Tampau y col. 2020). Esta última se considera una matriz prometedora y altamente viable ya que es un material relativamente económico, biodegradable, presenta una muy baja toxicidad y alta solubilidad en agua lo que lo convierte en un candidato idóneo para la formación de películas poliméricas con propiedades luminiscentes.

Por ejemplo, Jashobanta Sahoo y col. (2017) fabricaron con éxito películas de PVA dopadas con el complejo de coordinación denominado $\mathrm{EuL}^{\mathrm{R}} \mathrm{Cl}_{3}$ y $\mathrm{EuL}^{\mathrm{S}} \mathrm{Cl}_{3}$ donde $\mathrm{L}^{\mathrm{R}}$ y $\mathrm{L}^{\mathrm{S}}$ son ligandos de base de Schiff de 2,9-dialdehído 1,10-fenantrolina y (R) y (S) isómeros de 2-amino-3-fenilpropanol. Las cuales fueron caracterizadas para investigar sus propiedades fotoluminiscentes, haciendo mención que las matrices poliméricas tuvieron mejores resultados ópticos en comparación con los complejos que solo estaban en solución acuosa. La mejora de las propiedades luminiscentes se asoció a procesos de transferencia de energía intra e intermoleculares que potencializaron la transferencia de energía en el sistema. Por otro lado, observaron que la disposición completamente estática de los complejos dentro de la matriz afecta de manera positiva a la emisión, ya que favorece a la disminución de sitios que propicien la desactivación luminiscente. Por lo tanto, las matrices poliméricas dopadas con complejos de europio presentan un mayor rendimiento cuántico general.(Sahoo y col. 2017).

El diseño y preparación de películas basados en matrices de PVA ha sido de gran relevancia en los últimos años. Por esta razón, en la presente investigación se prepararon películas dopadas con el complejo Eu $\left(\mathrm{OOCC}_{6} \mathrm{H}_{5}\right)_{3}\left(\mathrm{H}_{2} \mathrm{O}\right)_{3}$ a diferentes concentraciones molares en alcohol polivinílico, empleando la técnica "tape-casting". Se evaluaron sus propiedades luminiscentes y se discute la interacción fisicoquímica que contribuyó al incremento de la intensidad luminiscente.

\section{Metodología}

\subsection{Materiales y métodos}

Se utilizó ácido benzoico (99\%), hidróxido de sodio $(99,9 \%)$ y cloruro de europio (III) (99\%) dichos reactivos fueron suministrados por Sigma-Aldrich (St. Louis, MI, EE. UU.), se utilizó agua desionizada como disolvente.

La espectrofotometría infrarroja por transformada de Fourier (FT-IR) se realizó con un equipo marca Perkin Elmer, modelo espectrómetro Spectrum 65 FT-IR en un rango de número de onda de 4000-400 $\mathrm{cm}^{-1}$. Las caracterizaciones de fotoluminiscencia de los complejos y de las películas dopadas se realizaron en un espectrofotómetro F-7000 FL marca HITACHI (High-Tech,Tokio,Japan), para el caso de las películas dopadas las caracterizaciones se realizaron a una longitud de excitación, $\lambda_{\mathrm{ex}}=290$ y monitorearon a una longitud de emisión, $\lambda_{\text {em }}=616$, para el caso del complejo EuL13 las 
caracterizaciones a una $\lambda_{\mathrm{em}}=616$ y una $\lambda_{\mathrm{ex}}=280$, para ambos casos se utilizó una apertura de rejilla de emisión y excitación de 2.5 .

\subsection{Síntesis del complejo Eu(OOCC $\left.6 \mathrm{H}_{5}\right)_{3} \cdot\left(\mathrm{H}_{2} \mathrm{O}\right)_{3}$}

Se preparó una disolución acuosa de hidróxido de sodio (1 mol. $\mathrm{L}^{-1}$ ) en donde posteriormente se disolvió $360 \mathrm{mg}$ de ácido benzoico con el fin de conseguir una desprotonación del ácido. De manera simultánea se preparó una disolución acuosa de cloruro de europio hexahidratado $\left(0.1 \mathrm{~mol} . \mathrm{L}^{-1}\right)$. Posteriormente, se adicionó la disolución de cloruro de europio a la del ácido benzoico en forma de goteo $\left(1 \mathrm{ml} \cdot \mathrm{min}^{-1}\right)$. La reacción se dejó reposar por $8 \mathrm{hrs}$. a condiciones ambientales manteniendo una agitación constante. Una vez transcurrido el tiempo de envejecimiento se retiró la reacción de la agitación y se prosiguió a realizar una serie de lavados de los polvos obtenidos utilizando papel filtro y agua desionizada con el fin de eliminar los excesos de impurezas solubles. Finalmente se dejó secar el producto a condiciones ambientales por $24 \mathrm{hrs}$.

\subsection{Preparación de películas de PVA}

Se prepararon suspensiones del complejo EuL3 a distintas concentraciones milimolares en $5 \mathrm{~mL}$ de agua desionizada (ver Tabla 1). Se dispersaron los polvos con ayuda de un baño ultrasónico comercial durante $10 \mathrm{~min}$. Posteriormente se colocó la suspensión en baño maría a una temperatura de $80{ }^{\circ} \mathrm{C}$. Una vez alcanzada la temperatura de $80{ }^{\circ} \mathrm{C}$ se incorporó un equivalente de $12 \% \mathrm{p} / \mathrm{p}$ de PVA con respecto al agua y se agitó de manera suave durante $30 \mathrm{~min}$ con el fin de disolver completamente el PVA. Pasado este tiempo se dejó enfriar por $1 \mathrm{~min}$, para finalmente colocar la mezcla en el equipo de recubrimientos "tapecasting" previamente calibrado a un espesor de $1 \mathrm{~mm}$ (La película se reduce a $0.5 \pm 0.03 \mathrm{~mm}$ después del secado) sobre un sustrato de papel Teflón® (la velocidad de recubrimiento fue de $0.5 \mathrm{~cm}$ por min). Las películas se dejaron secar a temperatura ambiente durante 24 hrs.

Tabla 1 Concentraciones molares de dopaje.

\begin{tabular}{llllll}
\hline Nomenclatura & EuL3-A & EuL3-B & EuL3-C & EuL3-D & EuL3-E \\
\hline $\begin{array}{l}\text { Cantidad de dopaje } \\
\text { de Eu }{ }^{3+}(\mathrm{mmol})\end{array}$ & 0.00026 & 0.00053 & 0.0011 & 0.0016 & 0.0021 \\
& & & & & \\
\hline
\end{tabular}

\section{Análisis de resultados y discusión}

\subsection{Análisis estructural de FT-IR}

En la Figura 1a se pueden distinguir distintas bandas de absorción asociadas a los enlaces característicos del PVA, por ejemplo, la banda localizada a $1096 \mathrm{~cm}^{-1}$ corresponde a la vibración por estiramiento del enlace C-O, a $2842 \mathrm{~cm}^{-1}$ y 2920 $\mathrm{cm}^{-1}$, podemos apreciar las bandas de vibración simétrica $\left(v_{\mathrm{sym}}\right.$ $\mathrm{C}-\mathrm{H})$ y asimétrica $\left(v_{\text {asym }} \mathrm{C}-\mathrm{H}\right)$ del enlace $\mathrm{C}-\mathrm{H}$ respectivamente, la banda de absorción que aparece a $3290 \mathrm{~cm}^{-1}$ pertenece a los grupos hidróxilos del PVA y moléculas de agua.

La Figura 1b muestra un acercamiento de región que va de

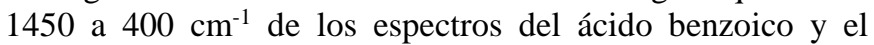
complejo EuL3, para el ácido benzoico se aprecia una banda
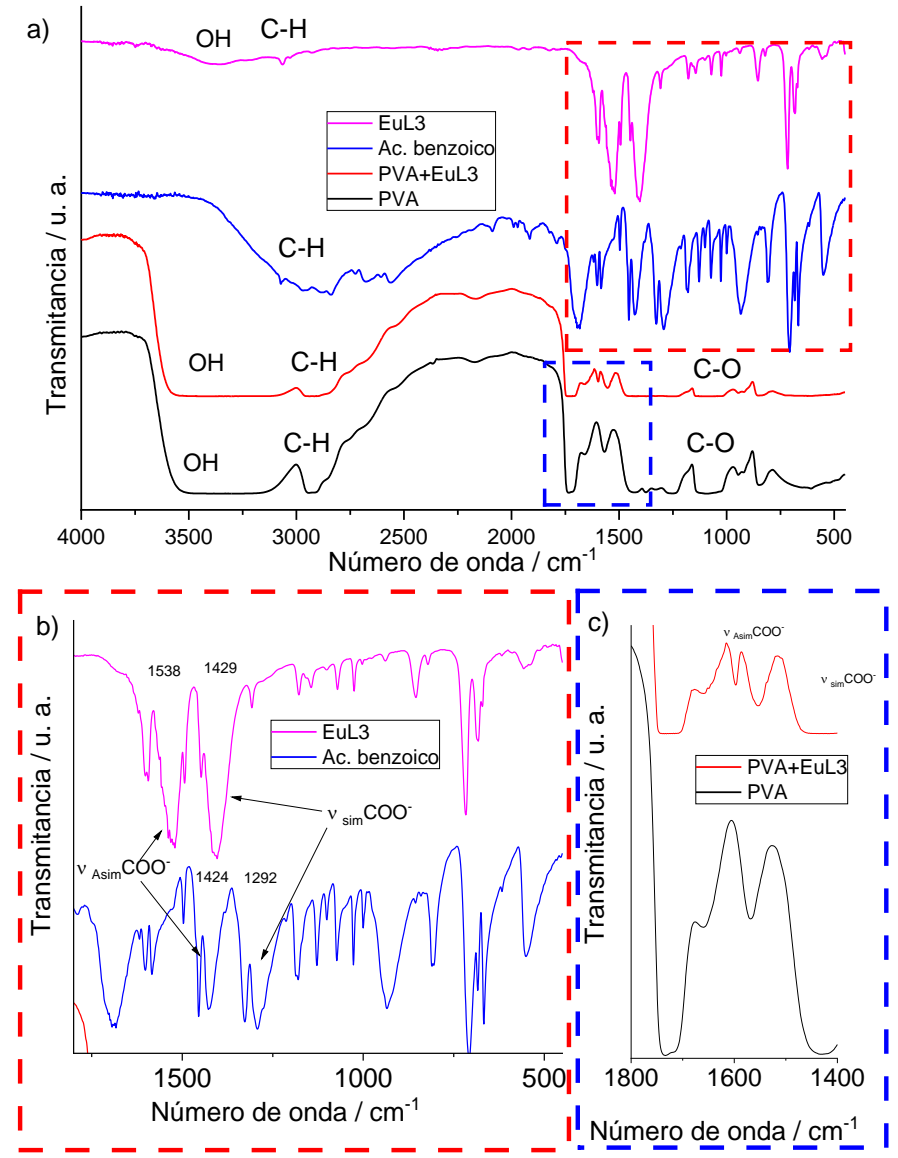

Figura 1: a) espectros IR de las películas, PVA, complejo y

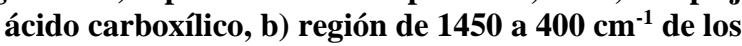
espectros del ácido benzoico y el complejo $\mathrm{EuL3}$, c) región de 1800 a $1400 \mathrm{~cm}^{-1}$ de los espectros del PVA y el sistema PVA+EuL3.

de absorción localizadas a $1421 \mathrm{~cm}^{-1}$ asociada a la vibración asimétrica del grupo carbonilo, $\left(v_{\mathrm{Asim}} \mathrm{COO}^{-}\right)$, y una a $1292 \mathrm{~cm}^{-1}$ correspondiente a la vibración simétrica del mismo grupo, $\left(v_{\text {Sim }} \mathrm{COO}^{-}\right)$. El complejo presenta un corrimiento de estas bandas a 1538 y $1429 \mathrm{~cm}^{-1}$ para $v_{\text {Asim }} \mathrm{COO}-E u$ y $v_{\text {Sim }} \mathrm{COO}-\mathrm{Eu}$, respectivamente. Esto último es el resultado del enlace grupo carbonilo con el núcleo de europio (Hern y col.2020). Las bandas antes mencionadas también se encuentran presentes en las películas dopadas (Figura 1c), por lo que se evidencia que el complejo está completamente embebido en la matriz y al no presentar ningún corrimiento significativo, de los enlaces característicos del complejo, por lo que añadimos que la estructura molecular del complejo se mantuvo integra en todo momento.

Jiangling He y col. mencionan que mientras el complejo de $\mathrm{Eu}^{3+}$ ingresa al polímero anfitrión, se espera que el ion $\mathrm{Eu}^{3+} \mathrm{se}$ coordine con los grupos polares del PVA. Tal interacción influye en la estructura local de la cadena principal del polímero y afecta a ciertos modos de vibración activos en donde la banda de absorción característica de los grupos $\mathrm{OH}$ (3368 $\mathrm{cm}^{-1}$ aprox.) tiende a ensancharse a medida que el ion $\mathrm{Eu}^{3+}$ se coordina al PVA, indicando de los grupos hidroxilo del PVA interactúan con los centros de $\mathrm{Eu}^{3+}$. (He y col., 2016) 
De igual manera Yifan Wei y col. (2020) reportan que la banda de absorción del enlace O-Eu debe de ser visible a 715 $\mathrm{cm}^{-1}$, pero en nuestro caso es complicado identificar dicha banda pues se encuentra completamente solapada por la banda del alcohol polivinílico (Wei y col.2020).

\subsection{Espectroscopia de luminiscencia}

En la Figura 2 se presenta el espectro de excitación del complejo de europio (EuL3) obtenido para una emisión máxima en $616 \mathrm{~nm}$ correspondiente a la transición ${ }^{5} \mathrm{D}_{0} \rightarrow{ }^{7} \mathrm{~F}_{2}$, se puede apreciar en primera instancia una banda ancha que va desde los 230-320 nm, alcanzando un máximo de excitación en $290 \mathrm{~nm}$. Esta transición se produce desde el estado excitado singlete de menor energía, $S_{1}$, hasta el estado fundamental $S_{0}$, por lo que es una transición $S_{0} \rightarrow$ $\mathrm{S}_{1}\left(\pi \rightarrow \pi^{*}\right)$, debido a que no se produce un cambio en la multiplicidad del estado. Esta transición está permitida por el espín, de forma que en ausencia de otros factores de simetría la emisión fluorescente está permitida y ese produce de manera rápida (en el rango de los picosegundos a los nanosegundos). A la derecha del espectro del complejo se aprecia las bandas de absorción que se originan desde el estado ${ }^{7} \mathrm{~F}_{0}$ hasta el estado excitado ${ }^{5} \mathrm{~L}_{7}$ centrado a $393 \mathrm{~nm}$, para el caso del sistema complejopolímero; estas últimas bandas se muestran con muy baja intensidad debido a los efectos de la matriz al interactuar con los núcleos de europio. Por lo tanto, esto sugiere una interacción entre ambas moléculas que produce que las transiciones $S_{0} \rightarrow S_{1}(\pi \rightarrow$ $\left.\pi^{*}\right)$, sean más representativas además de que muestra un desplazamiento de 290 a 280 nm en el pico máximo de absorción

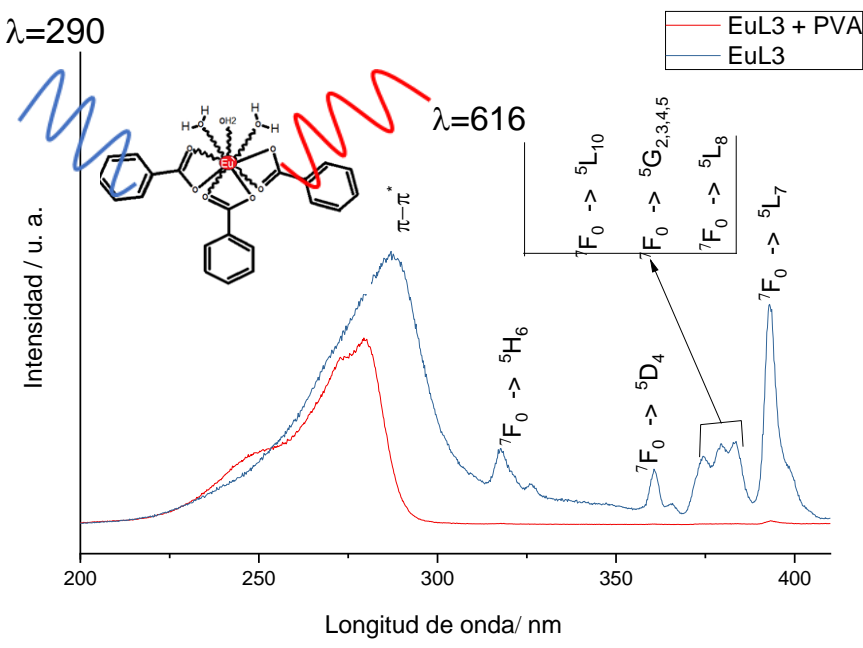

Figura 2: Espectro de excitación obtenido para una emisión de 616nm del complejo [EuL3] y su sistema complejopolímero.

En la Figura 3 se observa los espectros de emisión del complejo y las películas dopadas. Las bandas de emisión del mononuclear puro tanto para las películas dopadas se encuentra centradas a $\lambda_{\mathrm{em}}=616 \mathrm{~nm}$ y $592 \mathrm{~nm}$ pertenecientes a las tranciones ${ }^{5} \mathrm{D}_{0} \rightarrow{ }^{7} \mathrm{~F}_{2}$ producto a las transiciones dipolo eléctrico (DE) y ${ }^{5} \mathrm{D}_{0} \rightarrow{ }^{7} \mathrm{~F}_{1}$ producto de transiciones de tipo dipolo magnético (DM) respectivamente así mismo identificamos una disminución en la transición ${ }^{5} \mathrm{D}_{0} \rightarrow{ }^{7} \mathrm{~F}_{1}$ del DM que es una banda de emisión de color naranja brillante, independiente del ambiente o entorno en donde se encuentre el ion europio, mientras que la intensidad de la transición ${ }^{5} \mathrm{D}_{0} \rightarrow{ }^{7} \mathrm{~F}_{2}$ del DE muestra un aumento. En particular, las transiciones de ${ }^{5} \mathrm{D}_{0} \rightarrow{ }^{7} \mathrm{~F}_{2}$ se consideran una transición hipersensible es esta significativamente influenciada por el entorno de la matriz anfitrión alrededor de los iones $\mathrm{Eu}^{3+}$ (NarroGarcía y col.2015)

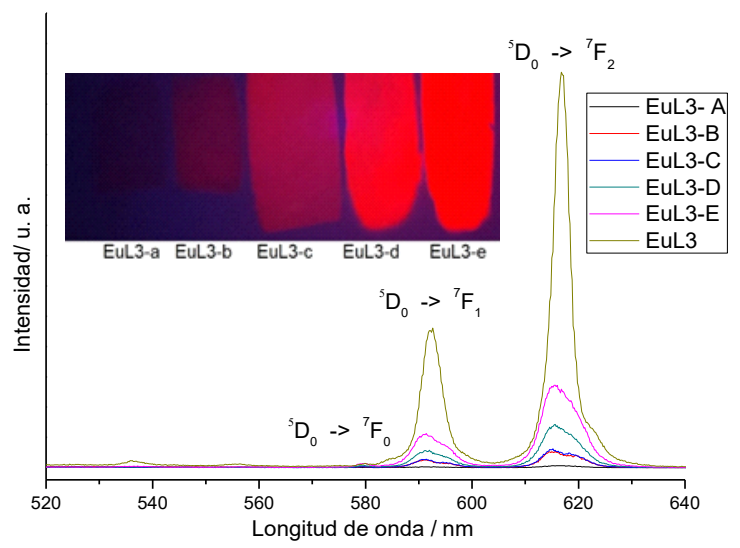

Figura 3: Espectro de emisión de las películas dopadas a una longitud de onda de excitación de $20 \mathrm{~nm}$.

Las intensidades de las transiciones de dipolos eléctricos podrían estar cambiando en función de las variaciones del centro de simetría debido a los cambios en el entorno del anfitrión alrededor del ion $\mathrm{Eu}^{3+}$. Por lo tanto, cuando los iones están situados en sitios de baja simetría, la transición DE tiene mayor probabilidad que la transición DM. Por el contrario, cuando los iones están situados en sitios simétricos, la transición DM tiene mayor probabilidad que la transición DE. La relación entre las intensidades de emisión a $616 \mathrm{~nm}\left({ }^{5} \mathrm{D}_{0} \rightarrow{ }^{7} \mathrm{~F}_{2}\right.$, DE) y $590 \mathrm{~nm}\left({ }^{5} \mathrm{D}_{0} \rightarrow{ }^{7} \mathrm{~F}_{1}\right.$, DM) podría aportar suficiente información para identificar el entorno de la matriz anfitrión alrededor de los iones $\mathrm{Eu}^{3+}$. Jiangling $\mathrm{He}$ y col. (2016) realizaron un experimento en donde comprobaron que moléculas de $\mathrm{EuCl}_{3}$ al estar en solución acuosa muestran un pico más alto a $590 \mathrm{~nm}$ que el pico a $616 \mathrm{~nm}$, lo que indica que el entorno alrededor de $\mathrm{Eu}^{3+}$ en solución acuosa es bastante simétrico. Mientras que a medida que una solución $\mathrm{Eu}^{3+} / \mathrm{PVA}$ se solidifica y resulta en la formación de una película, las intensidades de emisión en $616 \mathrm{~nm}$ se vuelven más altas que las de $590 \mathrm{~nm}$, lo que indica que el entorno alrededor de $\mathrm{Eu}^{3+}$ en matriz PVA se vuelve rígido y compacto genera sitios bastante asimétricos. En el caso de nuestras películas también podemos observar tal comportamiento de asimetría entre las bandas de DE y DM ya que de alguna manera la disposición estática de las moléculas propician el aumento en las transiciones ${ }^{5} \mathrm{D}_{0} \rightarrow{ }^{7} \mathrm{~F}_{2}(\mathrm{He}$ y col.., 2016). En la Figura 4 se aprecia una representación de la distribución de las moléculas de complejo a lo largo de las cadenas del PVA, siendo fuertemente influenciada por los sitios electronegativos de la cadena de PVA, Sahoo y col.( 2017), reportaron un comportamiento similar en la interacción entre complejos de $\mathrm{Eu}^{3+}$ al ser dispersados en una matriz de alcohol polivinílico, encontraron que la cadena macromolecular de cadena larga del PVA de la película en su estado sólido propicia un arreglo estático del complejo al crear una especie de arreglos moleculares ordenados, mientras que los respectivos centros $\mathrm{Eu}^{3+}$ facilitan así una estrecha proximidad entre sí. Proponiendo que tal disposición facilite la transferencia de energía de una molécula a otra y promueva la transferencia de energía intermolecular. También mencionan que durante esta transferencia de energía, la red utiliza una energía mínima y por lo tanto, la energía total transmitida al centro metálico aumenta, por otro lado también se menciona que otro factor que promueve el incremento en la 
transferencia de energía, se debe al fenómeno de apilamiento causado por grupos benceno pertenecientes a la molécula acomplejante (ligando) los cuales promueven que se genere un apilamiento intra e intermolecular del complejo en la matriz de PVA, este fenómeno es capaz de promover una transferencia de energía intramolecular que va desde el ligando hacia el centro metálico que traduce en un aumento significativo en la luminiscencia.

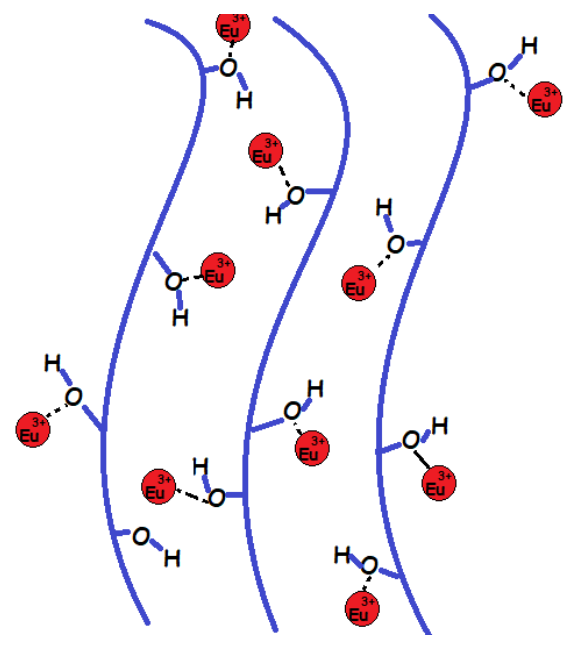

Figura 4: interpretación esquemática de las interacciones propuestas en formación del sistema PVA+EuLn3.

\section{Conclusiones}

Se sintetizaron películas de PVA dopadas con complejos de coordinación de $\mathrm{Eu}^{3+}$, por la técnica "Tape-Casting", la cual resulto ser una técnica de fácil manipulación y calibración, para la fabricación de nuestras películas. Las películas de PVA dopadas con el mononuclear tienen un desplazamiento en la banda de excitación sugiriendo una interacción entre los grupos más electronegativos de PVA $(\mathrm{OH})$ y el núcleo metálico. La interacción de PVA con el mononuclear alrededor de los iones. Incrementa la intensidad en la transición hipersensible ${ }^{5} \mathrm{D}_{0} \rightarrow{ }^{7} \mathrm{~F}_{2}$ del DE.

\section{Referencias}

Andreiadis, E. S., Gauthier, N., Imbert, D., Demadrille, R., Pécaut, J., \& Mazzanti, M. (2013). Lanthanide complexes based on $\beta$-diketonates and a tetradentate chromophore highly luminescent as powders and in polymers. Inorganic Chemistry, 52(24), 14382-14390. https://doi.org/10.1021/ic402523v

Binnemans, K. (2015). Interpretation of europium(III) spectra. Coordination Chemistry Reviews, 295, 1-45. https://doi.org/10.1016/j.ccr.2015.02.015

Byrne, J. P., Kitchen, J. A., Obrien, J. E., Peacock, R. D., \& Gunnlaugsson, T. (2015). Lanthanide directed self-assembly of highly luminescent supramolecular "peptide" bundles from $\alpha$-amino acid functionalized 2,6- bis(1,2,3-triazol-4-yl)pyridine (btp) ligands. Inorganic Chemistry, 54(4), 1426-1439. https://doi.org/10.1021/ic502384w

Chen, W., Li, M., Huibo, W., Zhiwei, L., Zuqiang, B., \& Chunhui, H. (2018). Advances in luminescent lanthanide complexes and applications. Science China Technological Sciences, 61(9), 1265-1285.

Crosby, G. A., Whan, R. E., \& Alire, R. M. (1961). Intramolecular energy transfer in rare earth chelates. Role of the triplet state. The Journal of Chemical Physics, 34(3), 743-748. https://doi.org/10.1063/1.1731670

Gibelli, E. B., Kai, J., Teotonio, E. E. S., Malta, O. L., Felinto, M. C. F. C., \& Brito, H. F. (2013). Photoluminescent PMMA polymer films doped with

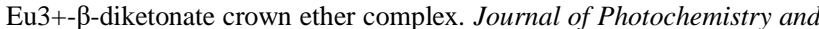
Photobiology A: Chemistry, 251, 154-159. https://doi.org/10.1016/j.jphotochem.2012.10.015

Gil-Kowalczyk, M., Łyszczek, R., Jusza, A., \& Piramidowicz, R. (2021). Thermal, spectroscopy and luminescent characterization of hybrid pmma/lanthanide complex materials. Materials, 14(12), 1-19. https://doi.org/10.3390/ma14123156

Gross, S., Camozzo, D., Di Noto, V., Armelao, L., \& Tondello, E. (2007). PMMA: A key macromolecular component for dielectric low- $\kappa$ hybrid inorganic-organic polymer films. European Polymer Journal, 43(3), 673696. https://doi.org/10.1016/j.eurpolymj.2006.12.012

He, J., He, Y., Zhuang, J., Zhang, H., Lei, B., \& Liu, Y. (2016). Luminescence properties of Eu $3 \mathrm{p} / \mathrm{CDs} / \mathrm{PVA}$ composite applied in light conversion fi lm. Optical Materials, 62, 458-464. https://doi.org/10.1016/j.optmat.2016.10.036

Hern, C., Ruiz-guerrero, R., \& Jes, A. De. (2020). New Mononuclear Complex of Europium (III) and Benzoic Acid: From Synthesis and Crystal. Iii.

Knyazev, A. A., Karyakin, M. E., Krupin, A. S., Romanova, K. A., \& Galyametdinov, Y. G. (2017). Influence of Eu(III) Complexes Structural Anisotropy on Luminescence of Doped Conjugated Polymer Blends. Inorganic Chemistry, 56(11), 6067-6075. https://doi.org/10.1021/acs.inorgchem.6b02825

LITTELL WC, \& JR. (1968). Use of Metal Masks To Improve Printing of Thick Film Geometrics. 123, 227-232. https://doi.org/10.1016/00262714(69)90347-3

Narro-García, R., Desirena, H., López-Luke, T., Guerrero-Contreras, J., Jayasankar, C. K., Quintero-Torres, R., \& De La Rosa, E. (2015). Spectroscopic properties of Eu3+/Nd3+ co-doped phosphate glasses and opaque glass-ceramics. Optical Materials, 46, 34-39. https://doi.org/10.1016/j.optmat.2015.03.051

Pham, Y. H., Trush, V. A., Amirkhanov, V. M., \& Gawryszewska, P. (2017). Structural and spectroscopic study of the europium complex with $\mathrm{N}$ (diphenylphosphoryl)pyrazine-2-carboxamide. Optical Materials, 74, 197-200. https://doi.org/10.1016/j.optmat.2017.04.031

Sahoo, J., lakshmi, D. S., Subramanian, P. S., George, T. M., \& Reddy, M. L. P. (2017). Synthesis, characterization and photo-physical properties of Eu(III) complexes and its luminescent thin films. Optical Materials, 70, 83-91. https://doi.org/10.1016/j.optmat.2017.05.015

Tampau, A., González-Martínez, C., \& Chiralt, A. (2020). Polyvinyl alcoholbased materials encapsulating carvacrol obtained by solvent casting and electrospinning. Reactive and Functional Polymers, 153(November 2019), 104603. https://doi.org/10.1016/j.reactfunctpolym.2020.104603

Wei, Y., Fu, Z., Zhao, H., Liang, R., Wang, C., Wang, D., \& Li, J. (2020). Preparation of PVA fluorescent gel and luminescence of europium sensitized by terbium (III). Polymers, 12(4). https://doi.org/10.3390/POLYM12040893

Xiang, S., Bao, D. X., Wang, J., Li, Y. C., \& Zhao, X. Q. (2017). Luminescent lanthanide coordination compounds with pyridine-2,6-dicarboxylic acid. Journal of Luminescence, 186, 273-282. https://doi.org/10.1016/j.jlumin.2017.02.037

Zhang, J., Zhang, L., Chen, Y., Huang, X., Wang, L., \& Zhang, Q. (2013). Influence of different carboxylic acid ligands on luminescent properties of $\mathrm{Eu}(\mathrm{Lc}) 3$ phen $(\mathrm{Lc}=\mathrm{MAA}, \mathrm{AA}, \mathrm{BA}, \mathrm{SA})$ complexes. Journal of Nanomaterials, 2013. https://doi.org/10.1155/2013/768535 